Positive Energy Density for the Alcubierre Warp Field Equations Using an RF-Driven Dielectric Resonant Cavity

This paper was downloaded from TechRxiv (https://www.techrxiv.org).

LICENSE

CC BY 4.0

SUBMISSION DATE / POSTED DATE

23-02-2022 / 03-03-2022

CITATION

Glenn, Chance (2022): Positive Energy Density for the Alcubierre Warp Field Equations Using an RF-Driven Dielectric Resonant Cavity. TechRxiv. Preprint. https://doi.org/10.36227/techrxiv.19224552.v1

DOI

10.36227/techrxiv.19224552.v1 


\title{
Positive Energy Density for the Alcubierre Warp Field Equations Using an RF-Driven Dielectric Resonant Cavity
}

\author{
Chance M. Glenn, Member, IEEE
}

\begin{abstract}
In this paper we show that the use of a resonant cavity filled with a lossy dielectric we can overcome the negative energy density requirement in Alcubierre's warp field equations. By using an imaginary part equal to or greater than the real part in the shaping function we generate a positive, non-exotic energy density requirement to generate and sustain the warp bubble necessary to transport a craft through space. We show simulation results for various shaping functions, the resulting Yorke time deformation, and the energy density over the warp bubble required. We discuss potential realizations of a device which generates such a field using electromagnetic and rf theory. Finally, we propose and show results of a simple experiment that uses these principles.
\end{abstract}

Index Terms-Alcubierre, warp bubble, spacetime, Yorke time, space travel, relativity, electromagnetics, resonance, dielectric.

\section{INTRODUCTION}

$\mathrm{I}$ $\mathrm{N}$ Alcubierre's seminal paper [1] he describes the possibility of moving a ship through by compressing spacetime in front of the craft and expanding it behind the craft, thus producing an apparent velocity, $v_{s}$, in a given direction. This warping of spacetime can produce apparent velocities that well exceed the speed of light yet does not violate Einstein's speed of light limit [2]. This has given many researchers and practitioners hope that we could build space vessels capable of reaching interstellar destinations within human lifetimes [3],[4],[5].

The Alcubierre metric is described in equation (1) and is a hyperbolic metric in $3+1$ space time. It is,

$d s^{2}=-d t^{2}+\left(d x-v_{s} f\left(r_{s}\right) d t\right)^{2}+d y^{2}+d z^{2}$.

The shaping function, $f\left(r_{s}\right)$, is an expression that describes the characteristics of the warp field that needs to be created to provide movement in the desired direction and is a function of the radius from the center of the ship. In equation (1) the shaping function influences the $x$ direction. Alcubierre offers a

This work was supported by a grant from the Morningbird Foundation. (Corresponding author: C. Glenn).

Chance M. Glenn, Sr. is professor with the University of Houston-Victoria, Victoria, TX 77901 USA (email: glennc1@uhv.edu) and a senior researcher with the Morningbird Foundation, Victoria, TX 77904 USA (email: chancemglenn@morningbirdfoundation.org). "top hat" shaping function described in equation (2). The function with respect to the radius is shown in fig. 1(a). The expression for the Yorke time expansion and contraction, $\theta\left(r_{s}\right)$, is in equation (3) wherein it is directly proportional to the derivative of the shaping function with respect to the radius. Fig. 1(b) shows the Yorke time response visually. What is desirable about the specific shaping function used is that it provides no space time distortion within a region where the ship can reside. Therefore, the passengers suffer no effects of time dilation or length contraction/expansion within that region [6].

$f\left(r_{s}\right)=\frac{\tanh \left(\sigma\left(r_{s}+R\right)\right)-\tanh \left(\sigma\left(r_{s}-R\right)\right)}{2 \tanh (\sigma R)}$

and

$\theta\left(r_{s}\right)=v_{s} \frac{x_{s}}{r_{s}} \frac{d f}{d r_{s}}$

In these expressions, $R$ is the radius of the warp bubble and $\sigma$ is the thickness of the warp bubble shell. There are no restrictions on the form of the shaping function, other than it would be beneficial to provide a region of unaffected spacetime wherein passengers can reside. As we will discuss in the next section, the required energy density is impacted by the shaping function.

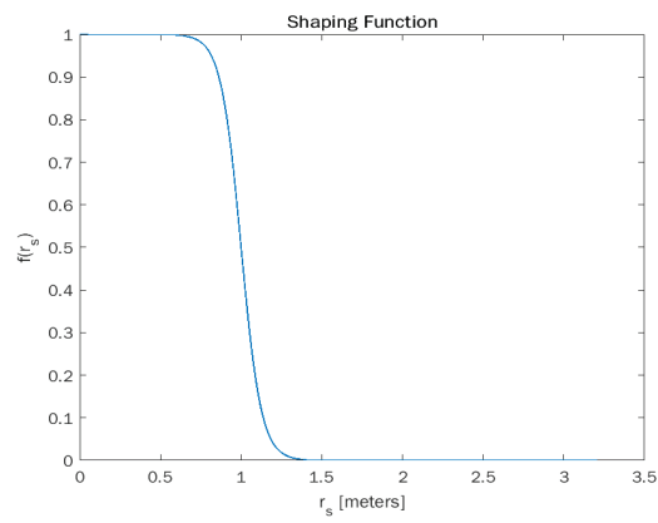

(a)

Color versions of one or more of the figures in this article are available online at http://ieeexplore.ieee.org 


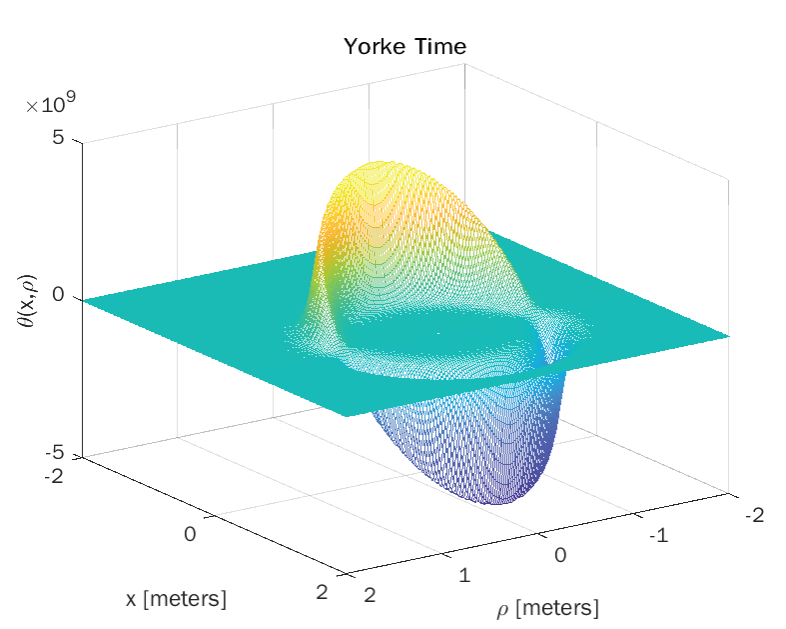

(b)

Fig. 1. Visualization of (a) the shaping function $f\left(r_{s}\right)$ and (b) the Yorke time contraction and expansion due to the shaping function provided in equation (2).

\section{ENERGY DENSITY}

The expression that is derived by Alcubierre for the required energy density is developed by calculating the Einstein tensor and is shown in equation (4). It provides negative values for the energy density which violates conditions for weak, dominant, and strong energy conditions as set forth by Hawking [7]. This suggests that the only way to form a warp bubble having any realizable shaping function is through the application of negative, or exotic, energy. While there has been much work engaged in reducing the resulting magnitude of this energy from the equivalent of mass contained within the entire known universe [8] to that of the mass of a small space vehicle [9], the solution is still a negative value. Some have even proposed the use of antimatter [10] and dark energy as a possible alternative [11], even though the existence of such an energy is currently theoretical at best [12].

$T^{00}=-\frac{1}{8 \pi} \frac{v_{s}^{2} \rho^{2}}{4 r_{s}^{2}}\left(\frac{d f}{d r_{s}}\right)^{2}$.

The energy density expression is proportional to the square of the derivative of the shaping function with respect to the radius. For the shaping function described in equation (2) the energy density is large and negative over the region containing the warp field. Figure 2 shows this. The total energy is found by integrating over the whole space.

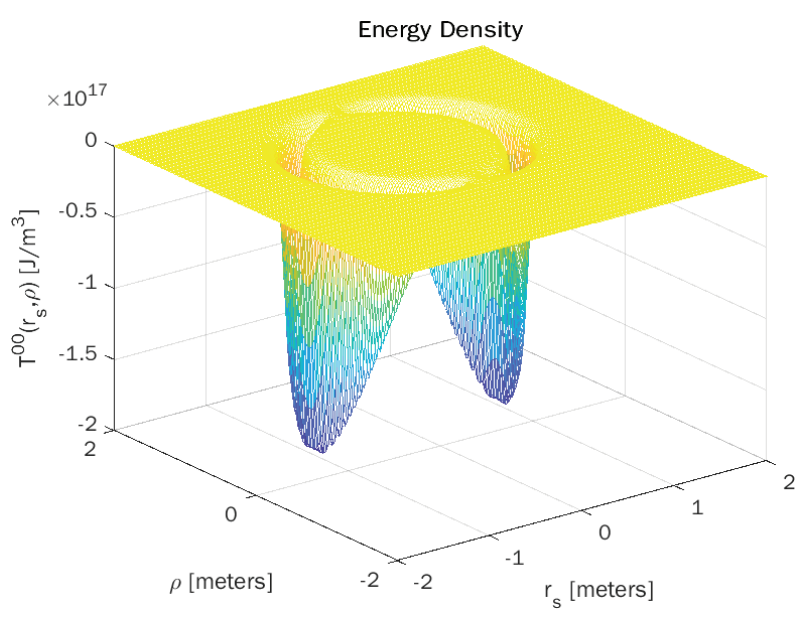

Fig. 2. Required energy density distribution for the shaping function described in equation (2).

In the next section we'll describe how we can create a shaping function that still maintains a region unaffected by Yorke time expansion and contraction yet allows the solution to the energy density equation to yield positive values.

\section{INTRODUCING A COMPLEX SHAPING FUNCTION}

If we solve equation (4) for $\frac{d f}{d r_{s}}$ we get,

$\frac{d f}{d r_{s}}=\sqrt{\frac{-32 \pi T^{00} r_{s}^{2}}{\rho^{2} v_{S}^{2}}}$,

which suggests that the derivative of the shaping function with respect to the radius must be complex if we are to have a positive energy density requirement. Suppose we allow the shaping function to have the following form,

$f_{c}\left(r_{s}\right)=f_{R}\left(r_{s}\right)+i f_{I}\left(r_{s}\right)=\gamma_{R} f\left(r_{s}\right)+i \gamma_{I} f\left(r_{s}\right)=\left(\gamma_{R}+\right.$

$\left.i \gamma_{I}\right) f\left(r_{s}\right)=\gamma f\left(r_{s}\right)$,

then,

$\frac{d f_{c}\left(r_{s}\right)}{d r_{s}}=\gamma \frac{d f\left(r_{s}\right)}{d r_{s}}$,

and

$$
\begin{aligned}
& \left(\frac{d f_{c}}{d r_{S}}\right)^{2}=\gamma^{2}\left(\frac{d f}{d r_{s}}\right)^{2} \\
& =\left(\gamma_{R}+i \gamma_{I}\right)^{2}\left(\frac{d f}{d r_{S}}\right)^{2} \\
& =\left(\gamma_{R}+i \gamma_{I}\right)\left(\gamma_{R}+i \gamma_{I}\right)\left(\frac{d f}{d r_{S}}\right)^{2} \\
& =\left(\gamma_{R}^{2}+i \gamma_{R} \gamma_{I}-\gamma_{I}^{2}\right)\left(\frac{d f}{d r_{S}}\right)^{2}
\end{aligned}
$$

So then, 
$\operatorname{Re}\left[\left(\frac{d f_{c}}{d r_{s}}\right)^{2}\right]=\left(\gamma_{R}^{2}-\gamma_{I}^{2}\right)\left(\frac{d f}{d r_{s}}\right)^{2}$

Therefore if $\left|\gamma_{I}\right|>\left|\gamma_{R}\right|$ then the square of the derivative of the shaping function with respect to the radius is negative, which allows the required energy density distribution to be positive, and perhaps even be in more of a manageable magnitude. Fig. 3(a) shows a map of the values of the energy density as the values of $\gamma_{R}$ and $\gamma_{I}$ vary while fig. 3 shows a graph of the value of the energy density given the ratio of $\gamma_{R}$ to $\gamma_{I}$. These relationships are calculated using the "top hat" shaping function first introduced by Alcubierre.

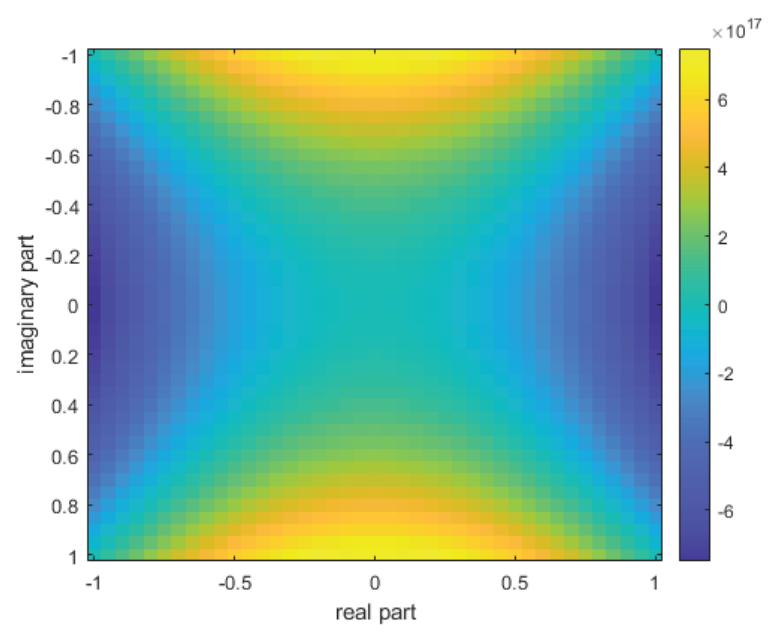

Fig. 3. A map of the total required energy given the values of the complex components $\gamma_{R}$ and $\gamma_{I}$.

Now if we select the values $\gamma_{R}=0.2$ and $\gamma_{I}=0.6$ then we see the energy density distribution shown in fig. 4 , which also provides the desired Yorke time structure as before, as shown in fig. 1(b).

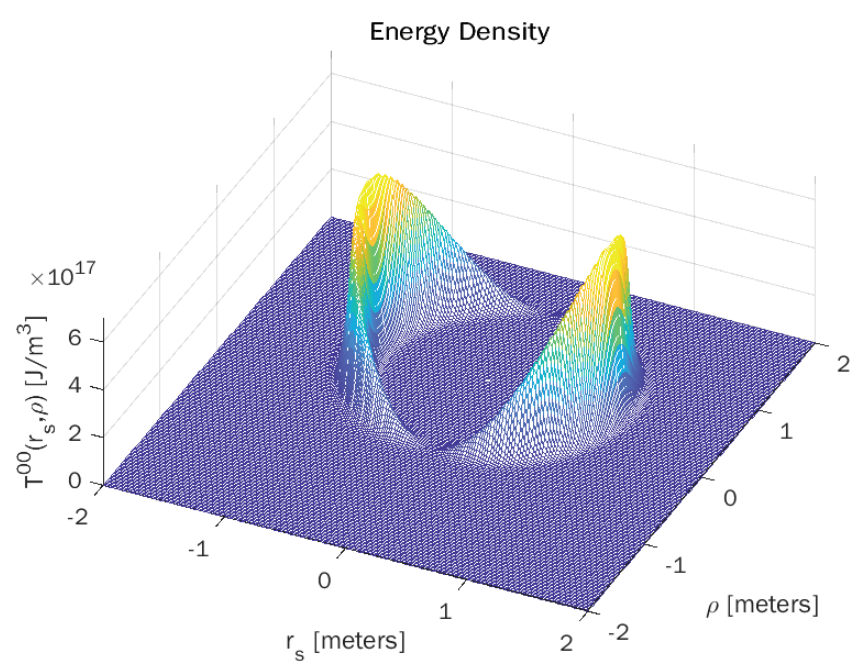

Figure 4. The energy density distribution for $\gamma_{R}=0.2$ and $\gamma_{I}$ $=0.6$.

\section{SIMULATION RESULTS}

The top hat shaping function that been proposed is ideal for creating a region of normal spacetime within the warp bubble. As White pointed out [13] the size and thickness of the warp bubble has a significant impact on the energy density requirement. In the following discussion we will look at three additional shaping functions and the energy required as a result. As we discuss realizations it will shed some light on the potential experimental results and the way that we might actually be able to produce a physical system.

In our simulation we will look at (1) a quarter cosine function whose peak is at the center of the ship position $\left(r_{s}=0\right)$ and is zero at $r_{s}=R$. We will look at (2) a cosine squared function that is zero at $r_{s}=R$, and (3) a $\sin x / x$ function that is damped as it approaches $r_{s}=R$. For each simulation the apparent velocity $v_{s}$ $=10 c$, the radius of the warp bubble is 1 meter, and the "ship" sits in the center of the warp field. For each situation we'll show the shaping function, the total energy over the complex plane, and the Yorke time distribution and the energy density for a specific set of complex values.

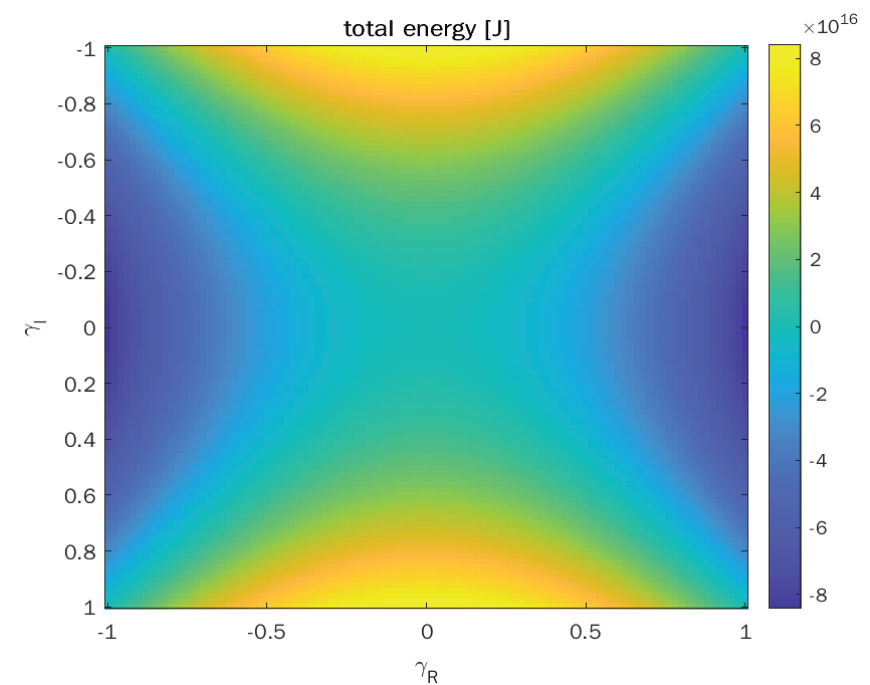

(a)

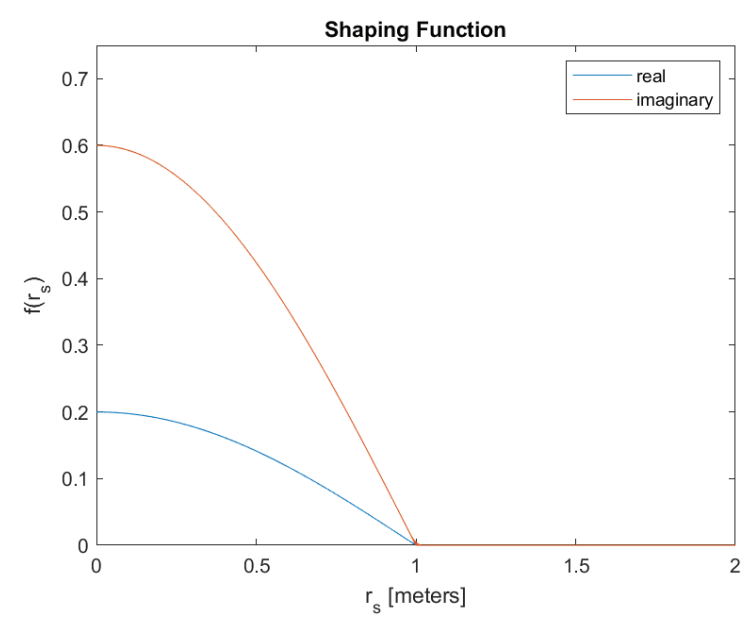

(b) 


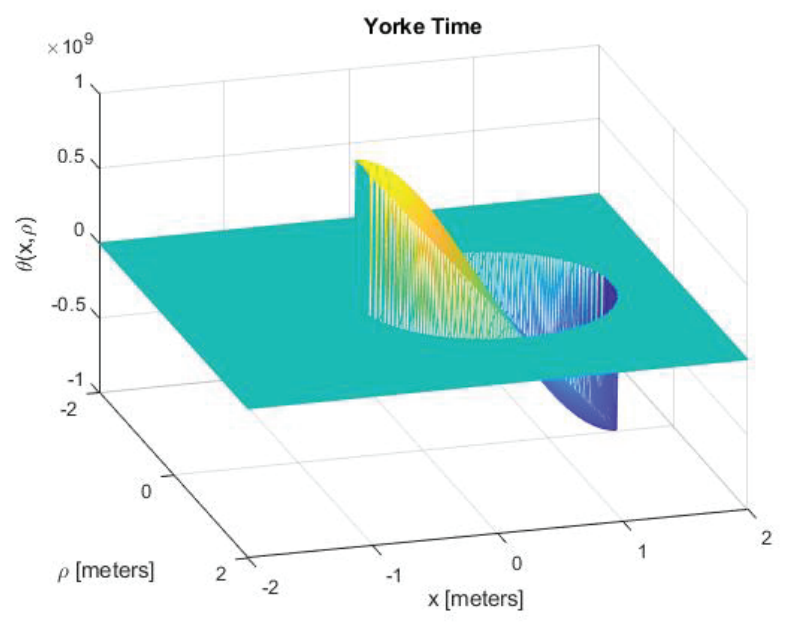

(c)

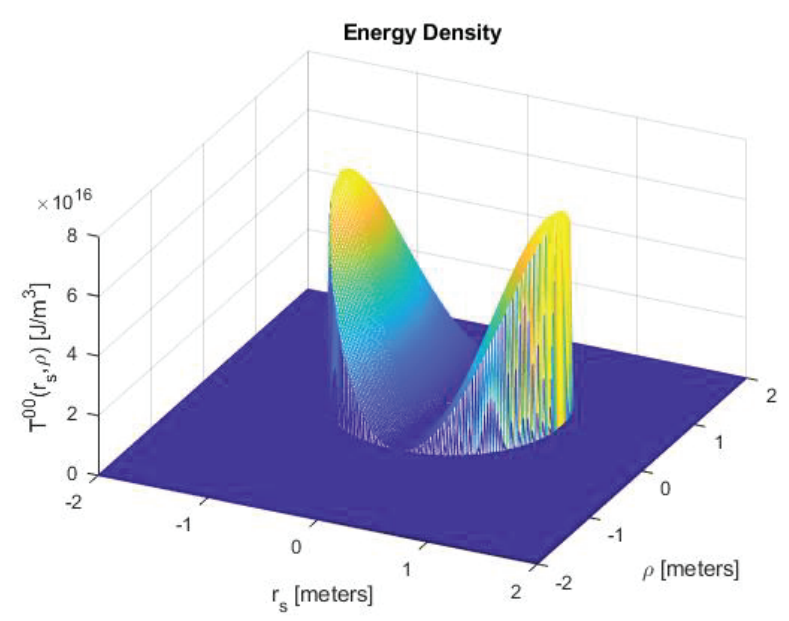

(d)

Fig. 5. The (a) total energy mapping over the complex plane, the (b) shaping function, (c) Yorke time distribution, and (d) energy density for $\gamma_{R}=0.2$ and $\gamma_{I}=0.6$ using a quarter cosine expression.

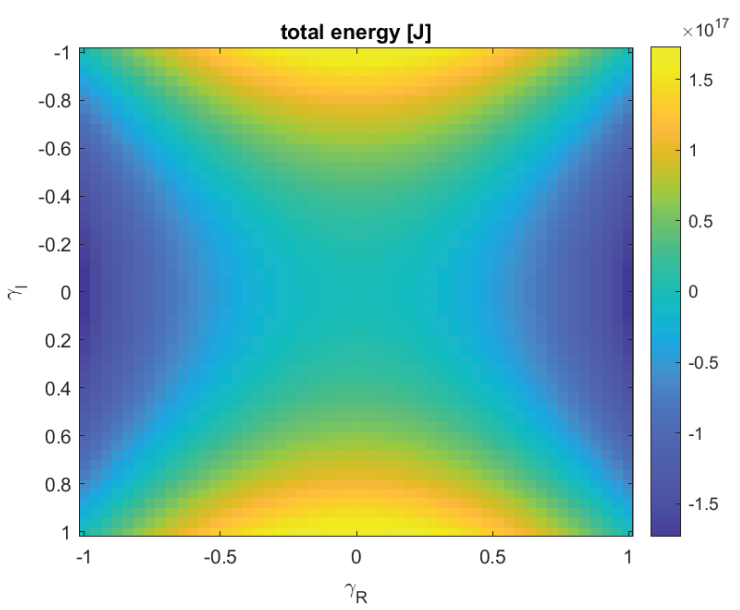

(a)

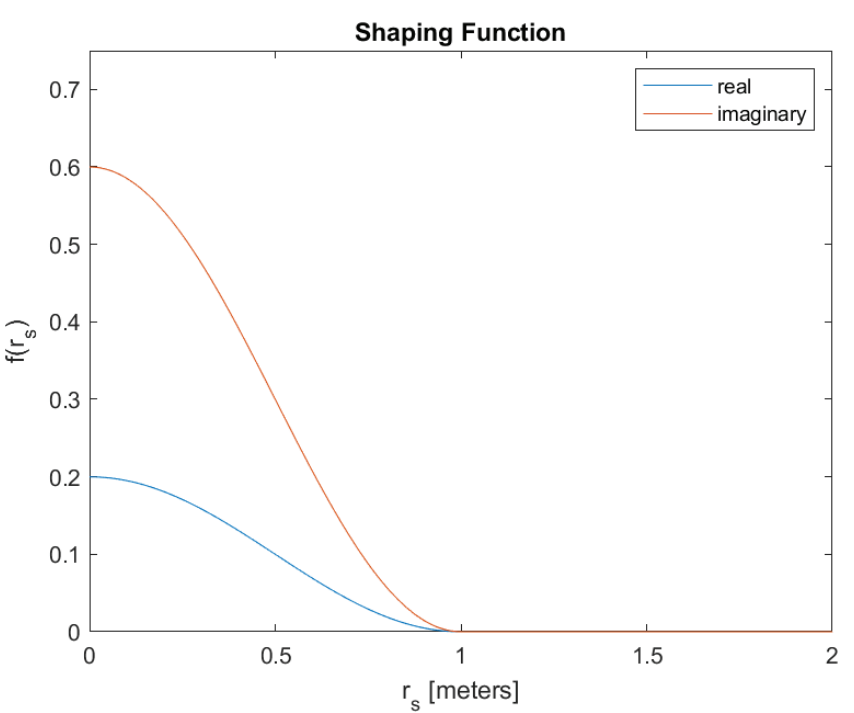

(b)

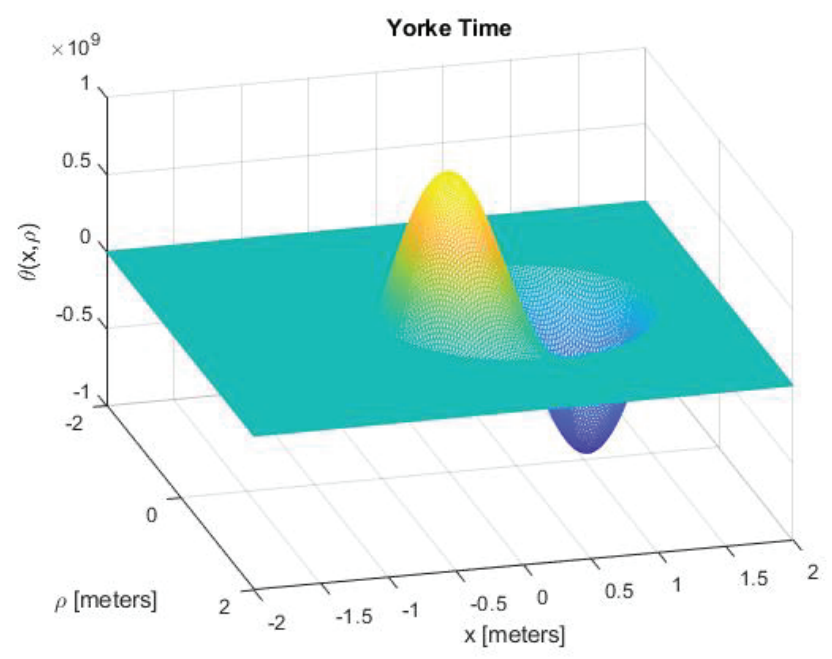

(c)

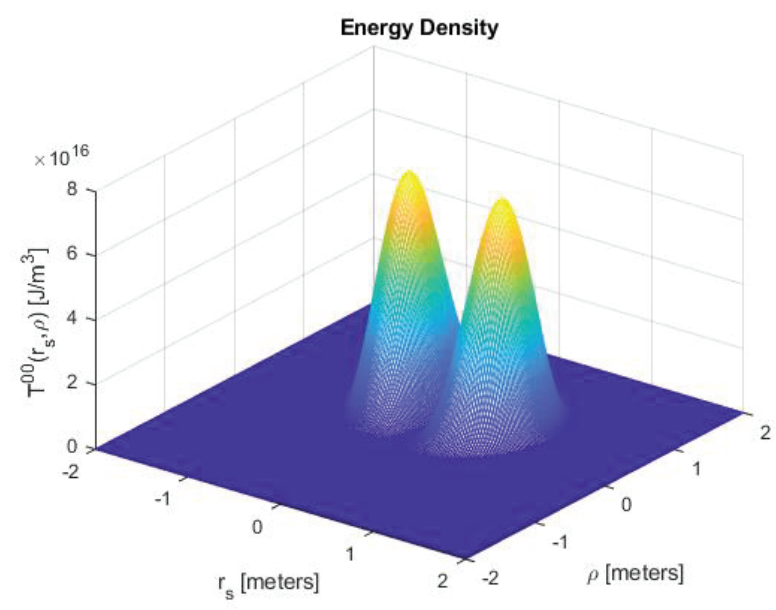

(d) 
Fig. 6. The (a) total energy mapping over the complex plane, the (b) shaping function, (c) Yorke time distribution, and (d) energy density for $\gamma_{R}=0.2$ and $\gamma_{I}=0.6$ using a cosine squared expression.

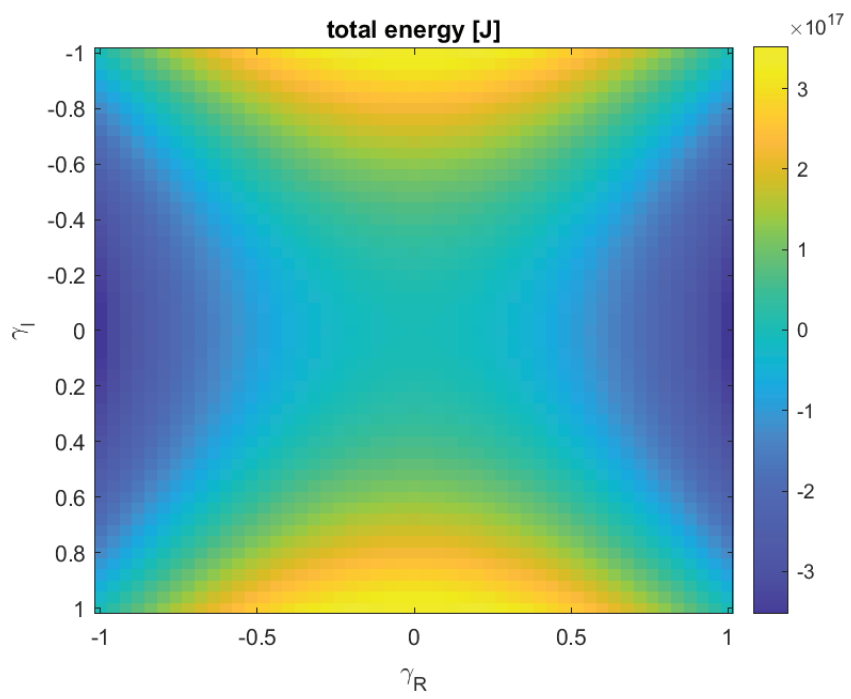

(a)

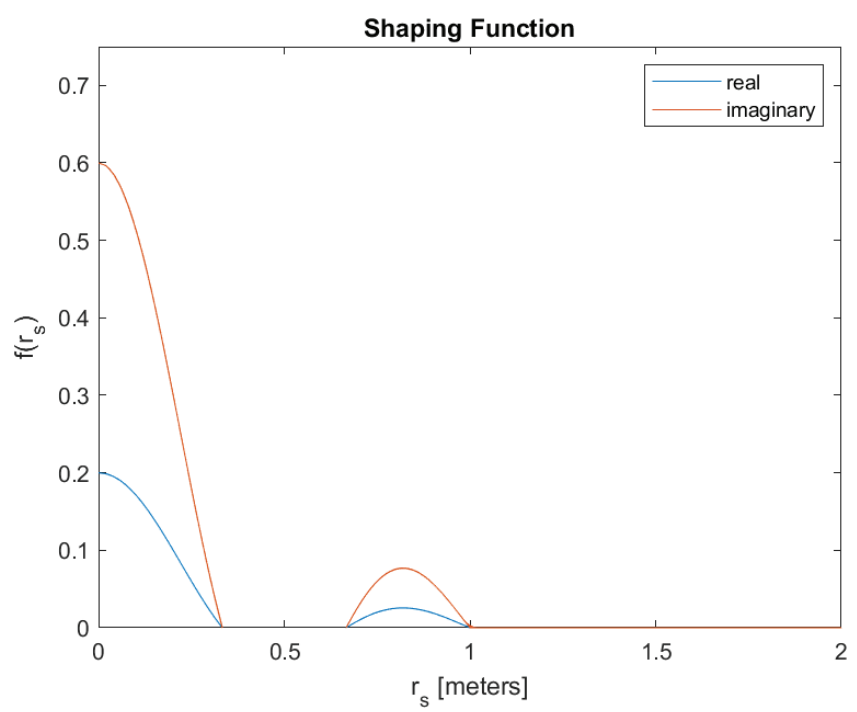

(b)

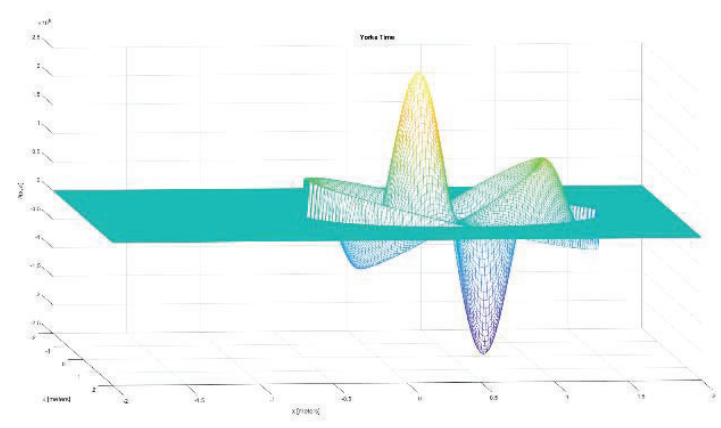

(c)

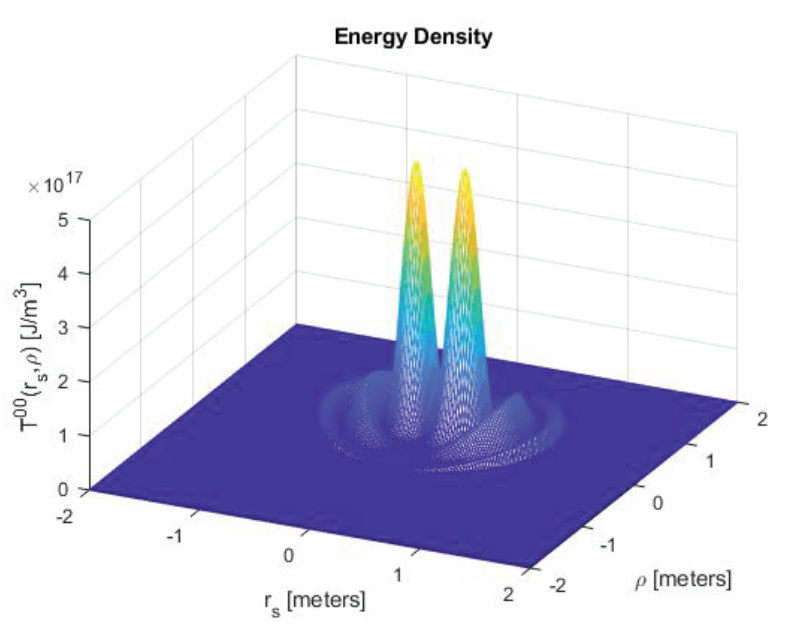

(d)

Fig. 7. The (a) total energy mapping over the complex plane, the (b) shaping function, (c) Yorke time distribution, and (d) energy density for $\gamma_{R}=0.2$ and $\gamma_{I}=0.6$ using a $\sin x / x$ expression.

\section{REALIZATION}

Einstein postulated that the gravitational pull between two bodies was due to the bending of space time that occurs due to the mass of the objects [2]. The Alcubierre metric was formulated from Einstein's gravitational field equation formalism. Furthermore, there exists a relationship between gravitational field equations and Maxwell's equations, which describe electromagnetic interactions [14]. Electromagnetism can be formulated mathematically in Minkowski space [15]. This suggests that electromagnetic fields have the potential to affect space time. The converse is clearly true as we are aware that strong gravitational fields bend light [16], which can be characterized as an electromagnetic wave.

A general solution to the wave equation, which comes from Maxwell's equations sets forth the expressions for the electric field $E$, as

$E(r, t)=E_{0} e^{i k r} e^{-i \omega t}$,

and the energy density due to the electric field is

$u=\varepsilon E^{2}$,

where $\varepsilon$ is the permittivity of the medium. Our strategy is to construct a "warp core" from a resonant cavity filled with a dielectric material with complex permittivity. That is,

$\varepsilon=\varepsilon^{\prime}+i \varepsilon^{\prime \prime}=\left(\varepsilon_{R}^{\prime}+i \varepsilon_{R}^{\prime \prime}\right) \varepsilon_{0}$.

Inside of the cavity, the shaping function is essentially the electric flux density $D=\varepsilon E$. Under a transverse magnetic mode, particularly $\mathrm{TM}_{010}$, the field strength is maximum at the 
$>$ REPLACE THIS LINE WITH YOUR MANUSCRIPT ID NUMBER (DOUBLE-CLICK HERE TO EDIT) <

center of the cavity. We have identified a liquid material that has the complex permittivity of $\varepsilon_{R}=12.21+14.52 i$ at a frequency of around $2.4 \mathrm{GHz}$ and a temperature of $20^{\circ} \mathrm{C}$ [18]. As we showed in the previous section, if the imaginary part of the shaping function is greater than the real part, then the energy density distribution required to distort spacetime (Yorke time) in the manner shown is positive.

Fig. 8 shows a diagram of the cylindrical cavity "warp core" to be used for the simulation and experiment. The diameter of the cylinder is calculated as a half wavelength in the material to be used. The phase velocity is calculated as $v=c / \sqrt{\varepsilon_{R}^{\prime}}$, so then $\lambda=\frac{v}{f}=\frac{c}{f \sqrt{\varepsilon_{R}^{\prime}}}=35.8 \mathrm{~mm}$. In order to establish a $\mathrm{TM}_{010}$ mode the radius of the cavity will be $8.9 \mathrm{~mm}$, and we use the condition that $\mathrm{d} / \mathrm{r}<2.03$, or $\mathrm{d}<18.09 \mathrm{~mm}$ [19]. Our goal is to demonstrate that a region of warped spacetime is created at the center of the cavity. The cavity is made such that there is a hole down the center which will allow a laser beam to pass through it through it unimpeded. If there is a perturbation of spacetime anywhere within this region, the laser beam will be slightly deflected.

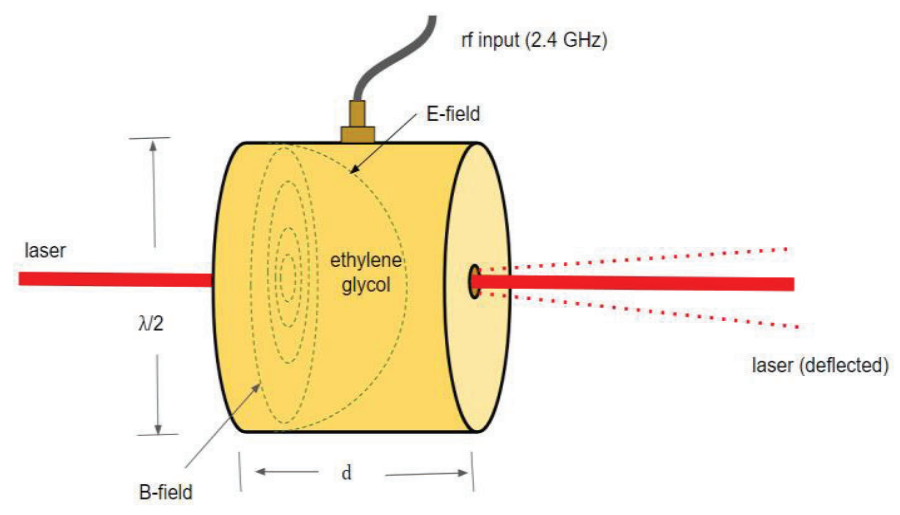

Fig. 8. Diagram of a cylindrical cavity resonator with $\mathrm{rf}$ at 2.4 $\mathrm{GHz}$ in $\mathrm{TM}_{010}$ mode used to perturb spacetime and deflect a laser beam passing through it.

Given the conditions set above, the shaping function, based upon the electric flux density has a $\cos (r)$ dependence as shown in fig. 9, with the real and imaginary parts of the function determined by the permittivity of the dielectric.

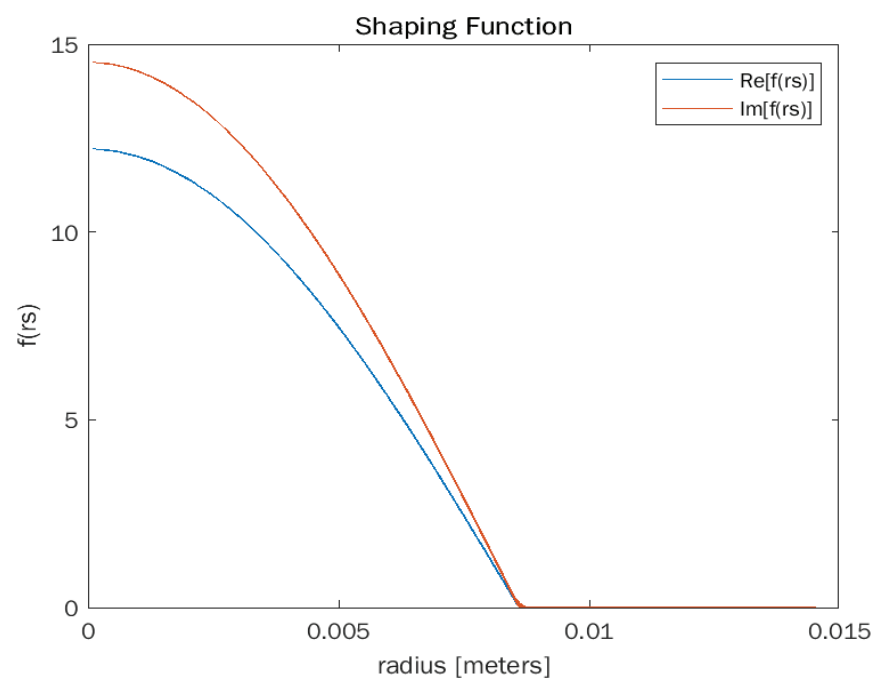

Fig. 9. The shaping function formed inside of the cylindrical cavity based upon the electric flux density in $\mathrm{TM}_{010}$ mode.

Under these conditions we expect the energy density to be that which is show in fig. 10(a). It is positive and highest on the outer edges of the radius. The Yorke time response in fig. 10(b) shows that in the region ahead of the "ship" spacetime is compressed and it is expanded in the region behind. There is only a negligible region where no spacetime compression or expansion occurs. The experimental process in this situation is such that we hope to observe some level of spacetime effect. Should this be effective then work can be done on the geometry or in the distribution of the dielectric material to produce a more useful shaping function.

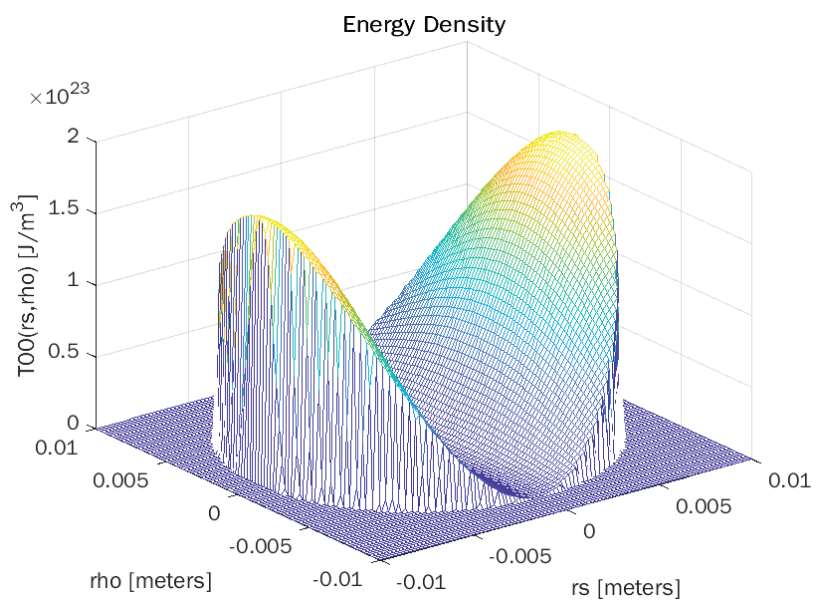

(a) 
$>$ REPLACE THIS LINE WITH YOUR MANUSCRIPT ID NUMBER (DOUBLE-CLICK HERE TO EDIT) <

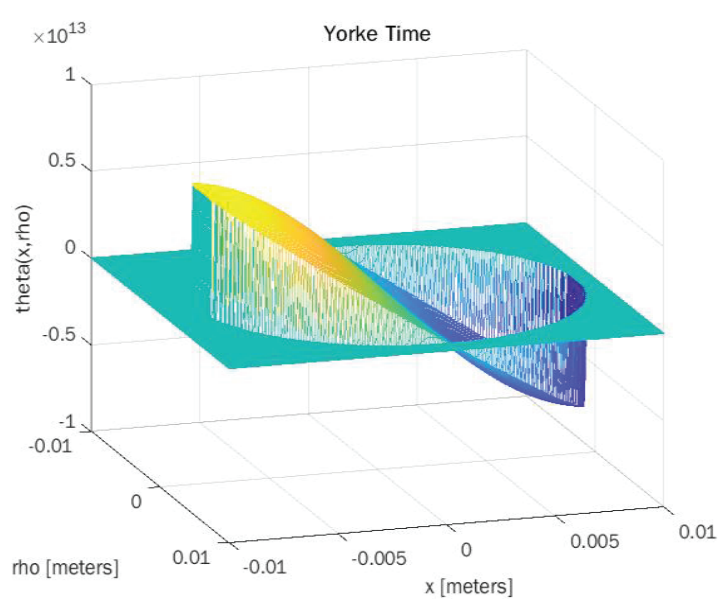

(b)

Fig. 10. The (a) energy density distribution and the (b) Yorke time structure based upon the cylindrical resonant cavity filled with a lossy dielectric excited with $2.4 \mathrm{GHz}$ in $\mathrm{TM}_{010}$ mode.

\section{EXPERIMENT}

Our goal is to determine if it is possible to initiate and detect any warping of space time by establishing an energy distribution within a confined space having a profile like what Alcubierre and White postulated. Any fluctuation, or distortion in space time would affect the trajectory of a beam of light that passes through that region. We set up a basic experiment to detect any deviation in the intensity or phase of a beam of light that passes through a region of a cavity having an $\mathrm{rf}$ field established in the manner simulated above. The cavity is such that the ethylene glycol is contained within it, yet there is a passthrough hole such that the laser beam is unimpeded. Fig. 11 shows a picture of the inside of this cavity having the probe required to introduce the rf into it and the complete cavity with an SMA connector.

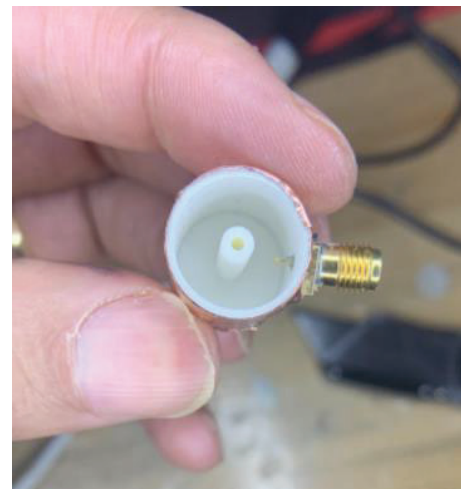

(a)

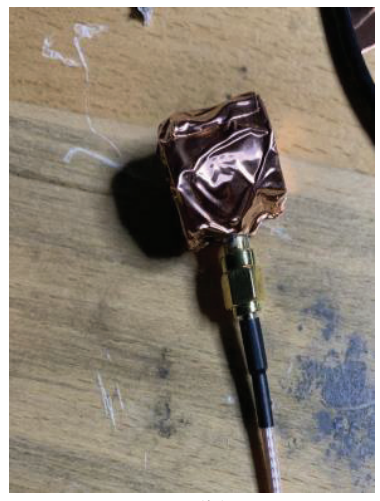

(b)
Fig. 11. (a)Inside of the $2.4 \mathrm{GHz}$ rf cavity which holds the ethylene glycol having a laser pass through hole, and (b) the complete enclosed "warp core".

\section{SETUP}

Fig. 12 shows a block diagram of the experiment setup. A $650 \mathrm{~nm}$ laser beam is passed through the rf cavity through the pass-through hole. It is captured by an optical sensor whose voltage is measured by an oscilloscope. The rf cavity is fed by a continuous $2.4 \mathrm{GHz}$ source at approximately $100 \mathrm{~mW}$ of power. Any fluctuations in voltage output to the scope will indicate that the laser beam has been affected by alteration in spacetime near it.

TABLE I

\section{RF CAVITY EXPERIMENT PARAMETERS}

Cylindrical cavity diameter:

$17.8 \mathrm{~mm}$

Pass through hole diameter

$1.5 \mathrm{~mm}$

rf source power:

$100 \mathrm{~mW}$

rf source frequency:

$2.4 \mathrm{GHz}$

Laser wavelength(s):

$410,532,650 \mathrm{~nm}$

Real Impedance in cavity:

$78 \mathrm{Ohms}$

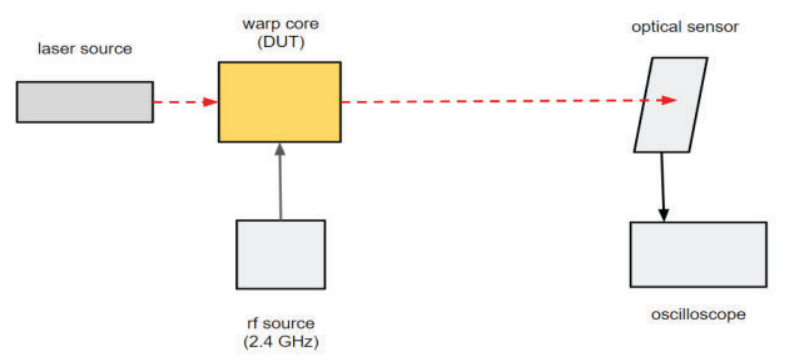

Fig. 12. Diagram of the experimental setup to test any spacetime variations produced by the "warp core".

\section{RESULTS}

After several attempts with three types of lasers, we observed no appreciable fluctuations in the voltage output on the oscilloscope when we introduced the rf into the dielectric filled cavity. This is not unexpected given that the energy densities required are several orders of magnitude higher than what we were able to produce with this bench top experiment.

A back of the envelope calculation of the power density produced starts by using known maximum power of the rf input to get the maximum voltage as,

$P_{\max }=\frac{V_{\max }^{2}}{Z_{0}} \rightarrow V_{\max }=\sqrt{P_{\max } Z_{0}}$

The maximum electric field in the cavity is $E_{\max }=\frac{V_{\max }}{d}$, where $d=\frac{\lambda}{2}$. Inside the cavity, with it filled with ethylene glycol, $\lambda=$ $\frac{v}{f}=\frac{1}{f \sqrt{\varepsilon \mu}}=\frac{c}{f \sqrt{\varepsilon_{r} \mu_{r}}}=17.2 \mathrm{~mm}$, so $\lambda / 2=8.6 \mathrm{~mm}$. Therefore,

$E_{\text {max }}=\frac{2 V_{\max }}{\lambda}=\frac{2 \sqrt{P_{\max } Z_{0}}}{\lambda}$.

The energy density in the cavity due to the rf is

$u_{E}=\varepsilon E^{2}=\varepsilon\left(\frac{2 \sqrt{P_{\max } Z_{0}}}{\lambda}\right)^{2}=7.3 \mu \mathrm{J} / \mathrm{m}^{2}$ 
which is far short of the required energy density levels to distort spacetime. As the goal here is to initiate the warping of spacetime in a local region it would be advantageous to determine a minimum required energy density to do so. Obousey [11] proposes a minimal energy density requirement based on the Hubble constant but like White [9], suggests that this extremely high value can be reduced by thinner walls at the edge of the warp bubble. We also recommend pulsed power in order to establish a higher differential energy with respect to the radius. In addition, if higher input energy is concentrated over a smaller region then the energy density is higher.

\section{SUMMARY}

We have established mathematically that there exists the potential to realize a positive required energy density in order to warp space time. By using a complex shaping function where the imaginary part is greater than the real part, the solution to the expression for the required energy density is positive. This ultimately makes it feasible to selectively bend spacetime for the purposes of propelling a craft.

We have devised an experiment that utilizes a material having the properties of possessing a greater imaginary component to the dielectric constant than real component. This material, introduced into a resonant cavity, sets up the conditions to realize a shaping function in the form as is utilized in the Alcubierre's metric. While we were not able to detect a distortion to spacetime we have established a potential structure from which to build better experiments.

Additional considerations are to utilize metamaterials within an rf chamber or cavity for the establishment of a complex structure. By setting the stage for a positive energy density to warp space time we move much closer to realizing this extraordinary technological capability.

\section{ACKNOWLEDGMENT}

Chance M. Glenn thanks Dr. Charles Westgate for guidance and insight for this work and work over the years. He would also like to thank the leadership of the Morningbird Foundation for support throughout the course of this endeavor.

\section{REFERENCES}

[1] Alcubierre, M., "The warp drive: hyper-fast travel within general relativity," Class. Quant. Grav. 11, L73-L77 (1994).

[2] Einstein, A., \& Lawson, R. W. (1921). Relativity: The special and general theory. New York: Holt.

[3] White, H., Davis, E., "The Alcubierre Warp Drive in Higher Dimensional Space-time," in proceedings of Space Technology and Applications International Forum (STAIF 2006), edited by M. S. ElGenk, American Institute of Physics, Melville, New York, (2006).

[4] Chung, D. J. H., and Freese, K., "Can geodesics in extra dimensions solve the cosmological horizon problem?", Phys. Rev. D 62, 063513 (2000).

[5] Ellis, George F. R.; Williams, Ruth M. (2000). Flat and Curved Spacetimes (2n ed.). Oxford University Press. pp. 28-29.

[6] Alcubierre, M., Alcubierre Lecture: Starship Congress 2017: Miguel Alcubierre, "Faster Than the Speed of Light" https://youtu.be/5q_z8BjiYng.

[7] S.W. Hawking and G.F.R. Ellis, The Large-Scale Structure of Spacetime, Cambridge University Press, (1973).

[8] Gast, Robert. "Star Trek's Warp Drive Leads to New Physics". Scientific
American. Retrieved 21 December 2021.

[9] White, H., "A Discussion on space-time metric engineering," Gen. Rel. Grav. 35, 2025-2033 (2003).

[10] Farnes, J.S. (2018). "A Unifying Theory of Dark Energy and Dark Matter: Negative Masses and Matter Creation within a Modified $\Lambda \mathrm{CDM}$ Framework". Astronomy \& Astrophysics. 620: A92.

[11] Obousey, R. "Warp Drive, Dark Energy, and the Manipulation of Extra Dimensions", DiA-08-1004-001, April 2010.

[12] Peebles, P. J. E.; Ratra, Bharat (2003). "The cosmological constant and dark energy". Reviews of Modern Physics. 75 (2): 559-606.

[13] White, H. "Warp Field Mechanics: 101: Energy Optimization", Presentation, Johnson Spaceflight Center.

[14] David Delphenich (2015). "Pre-metric electromagnetism as a path to unification". Unified Field Mechanics: Natural Science Beyond the Veil of Spacetime, Morgan State University, USA, 16-19 November 2014: 215-220.

[15] Minkowski, Hermann (1907-1908), "Die Grundgleichungen für die elektromagnetischen Vorgänge in bewegten Körpern" [The Fundamental Equations for Electromagnetic Processes in Moving Bodies], Nachrichten von der Gesellschaft der Wissenschaften zu Göttingen, Mathematisch-Physikalische Klasse: 53-111.

[16] Tilman Sauer (2008). "Nova Geminorum 1912 and the Origin of the Idea of Gravitational Lensing". Archive for History of Exact Sciences. 62 (1): $1-22$.

[17] Ikyumbur et al.; CJAST, 32(5): 1-7, 2019; Article no. CJAST.38947.

[18] Balanis, C. A. (1989). Advanced engineering electromagnetics. New York: Wiley.

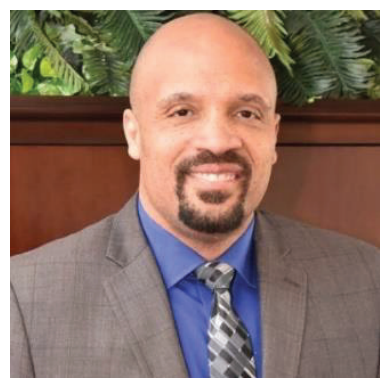

Chance M. Glenn (Member, IEEE) was born in Newark, NJ (USA) and is a professor of electrical engineering and an inventor. He holds a Ph.D. and Master's degree in electrical engineering from the Johns Hopkins University Whiting School of Engineering (2003, 1995) in Baltimore, MD (USA) and a B.S. degree in electrical engineering from the University of Maryland (1991) in College Park, MD (USA).

$\mathrm{He}$ is currently Provost and Vice President of Academic Affairs at the University of Houston-Victoria in Victoria, TX (USA). He is also the founder and senior researcher at the Morningbird Foundation in Victoria, TX (USA). He is published internationally, holds patents, and has conducted funded research and development in electromagnetics and rf, engineering applications of chaos theory, and advanced additive manufacturing and materials engineering.

Dr. Glenn is a member of the IEEE (Jan 2001) and was inducted into the Indispensable Role of Blacks in Johns Hopkins University in 2008, and holds memberships in the American Association for the Advancement of Science, the SPIE International Society for Photonics and Optics, the New York Academy of Sciences, the American Association of University Professors, the American Society of Engineering Education, the National Society of Black Engineers, the International Association for the Development of Advances in Technology, the National Academy of Recording Arts and Sciences, and the American Writers Guild East. He is also a member of the National is on the Board of Directors of the Starlink Initiative for SpaceX. 